\title{
Diblock copolymer membranes investigated by single-particle tracking $\dagger$
}

\author{
Chandrashekara R. Haramagatti, ${ }^{a}$ Felix H. Schacher, $\ddagger^{b}$ Axel H. E. Müller ${ }^{b}$ and \\ Jürgen Köhler*a
}

Received 31st August 2010, Accepted 29th October 2010

DOI: $10.1039 / \mathbf{c 0 c p 0 1 6 5 8 f}$

We report a study on particle diffusion in membranes formed from polystyrene-blockpoly(2-(dimethylamino)ethyl methacrylate) (PS- $b$-PDMAEMA) diblock copolymers.

The membranes were investigated by scanning electron microscopy and by single-particle tracking employing carboxy-functionalized polystyrene beads loaded with a fluorophore as spectroscopic probes. From the diffusion trajectories we extracted the domain size distribution of the membranes and the local diffusion coefficient of the beads as a function of the size of the beads. The single-particle tracking data revealed that the effective domain sizes of the membranes are reduced with respect to the domain sizes obtained from scanning electron microscopy, reflecting the confined diffusion of the probe particles due to interactions with the domain walls. This is corroborated by a clear correlation between the diffusion coefficient of an individual polystyrene bead and the size of the actual domain to which it is confined.

\section{Introduction}

The search for novel synthetic materials that can serve as membranes is still an issue of outstanding relevance, since such structures bear tremendous potential for technological applications in diverse fields such as catalysis, ${ }^{1,2}$ filtration and molecular sieving, ${ }^{3-5}$ chromatography,${ }^{6,7}$ dialysis, ${ }^{8,9}$ and the development of novel drug-delivery systems. ${ }^{10,11}$ General methods for the characterization of mesoporous structures with high spatial resolution are scanning- and/or transmission electron microscopy (SEM/TEM), or X-ray diffractometry (XRD). However, electron microscopy is highly invasive, demanding dry samples under vacuum conditions for imaging. Some polymers quickly degrade when subjected to the electron beam in SEM or TEM. XRD only provides information on periodic structures. In order to obtain information about the transport of guest molecules through the domains, neutron scattering, ${ }^{12,13}$ pulsed field-gradient nuclear magnetic resonance (NMR), ${ }^{14,15}$ or fluorescence-correlation spectroscopy (FCS) ${ }^{16,17}$ have been exploited. Yet, all these methods provide data that are averaged over many particles, leaving it extremely difficult to extract information about local heterogeneities of the membranes. Moreover, despite the high temporal resolution that is provided by FCS, this method is limited to short observation times, typically in the order of some milliseconds,

\footnotetext{
${ }^{a}$ Experimental Physics IV and Bayreuth Institute for Macromolecular Research (BIMF), University of Bayreuth, D-95440, Bayreuth, Germany.E-mail: juergen.koehler@uni-bayreuth.de;

Fax: + 49 921-55 4002; Tel: + 49 921-55 4001

${ }^{b}$ Macromolecular Chemistry II and Bayreuth Institute for

Macromolecular Research (BIMF), University of Bayreuth,

D-95440, Bayreuth, Germany

$\dagger$ Electronic supplementary information (ESI) available: Movies showing the confined diffusion of $20 \mathrm{~nm}$ polystyrene beads and SEM images of the membrane (bottom view \& cross section). See DOI: $10.1039 / \mathrm{c} 0 \mathrm{cp} 01658 \mathrm{f}$

$\ddagger$ Present address: Institute of Organic and Macromolecular Chemistry, Friedrich Schiller University Jena, D-07743 Jena, Germany.
}

which is determined by the time it takes for a fluorescent particle to move through the excitation volume.

An alternative approach to obtain information about porous materials on a truly local scale is provided by singleparticle tracking (SPT) ${ }^{18-24}$ There, the emission from an individual fluorescent particle is used to form a diffractionlimited image on an observation screen and the spatial position of the particle is reconstructed from the location of the maximum of the centroid of this image. ${ }^{25}$ This allows to determine the spatial position of the particle with an accuracy far beyond the classical diffraction limit of light microscopy and to follow the diffusion of this particle with high precision. ${ }^{26}$ This method has been exploited successfully to follow the diffusion of individual chromophores in lipid bilayers, ${ }^{27}$ or by recording the trajectories of the diffusion pathways of individual fluorescent probes, to elucidate local structural heterogeneities in silica mesostructures. ${ }^{22,28,29}$

A promising class of materials for membrane applications is block copolymers. Due to their self-assembly properties, which are based on the phase segregation of incompatible blocks, these materials allow to adjust or even to switch the morphology of the assembly on the nanoscale as a function of external stimuli. ${ }^{30}$ Moreover, the large number of end groups of these polymers can be functionalized, resulting in highly specific coatings, which might become of great importance for sensor applications or for specific binding of biomolecules. Here we report about a study on membranes formed from polystyrene-block-poly(2-dimethylaminoethyl methacrylate) (PS- $b$-PDMAEMA (Fig. 1)). The PDMAEMA block is double stimuli-responsive. Its solubility in water depends on the solution $\mathrm{pH}$ and on the temperature. Under acidic conditions $\left(\mathrm{pH} \ll \mathrm{p} K_{\mathrm{a}} \approx 7.7\right)^{31}$ the polymer chains are fully protonated and well dissolved and stretched, whereas a basic environment $\left(\mathrm{pH}>\mathrm{p} K_{\mathrm{a}}\right)$ causes a partial chain collapse. Increase of temperature leads to a full collapse, due to a $\mathrm{pH}$-dependent Lower Critical Solution Temperature (LCST). ${ }^{3,31}$ We investigated the structure of the membranes by SEM and SPT employing 

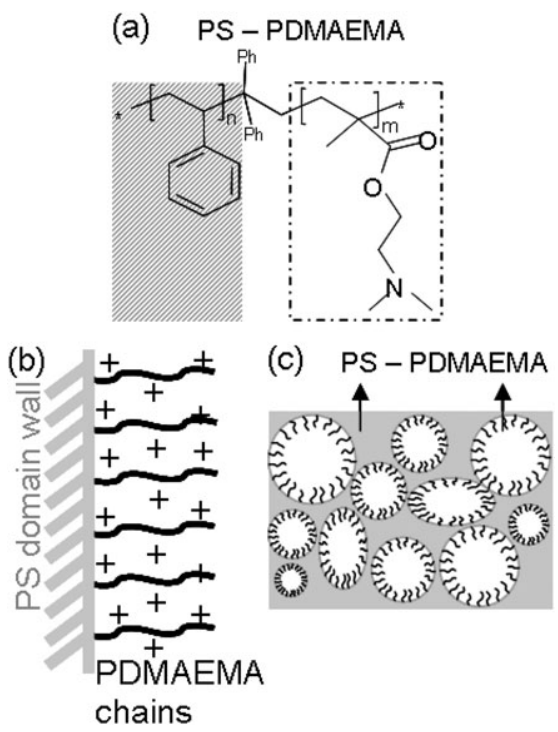

Fig. 1 (a) Molecular structure of the diblock copolymer polystyreneblock-poly(2-dimethylaminoethyl methacrylate) (PS-PDMAEMA), the PS block and the PDMAEMA chain are indicated by the shaded area and the dashed box, respectively. (b) Schematic sketch of the PDMAEMA chains attached to the domain walls. (c) Schematic sketch of the membrane. The PS block forms the bulk material (grey), and the insides of the domains (white) are covered with the PDMAEMA chains.

polymer beads of different sizes ranging from $20-500 \mathrm{~nm}$ in diameter and compared the domain size distribution of the membranes and the local diffusion coefficients as a function of the size of the beads and the diameters of the domains. The single-particle tracking data revealed that the effective domain sizes of the membranes are reduced with respect to the domain sizes obtained from scanning electron microscopy which presumably reflects the confined diffusion of the probe particles due to interactions with the domain walls. This is corroborated by a clear correlation between the diffusion coefficient of an individual polystyrene bead and the size of the actual domain to which it is confined. These findings are interesting not only for issues that touch on material transport through nanoporous membranes, a generic feature for the function of a membrane, but also for studying fundamental aspects of wall effects in hydrodynamic flow on the microscale.

\section{Experimental}

\section{Sample preparation}

The block copolymer polystyrene-block-poly(2-dimethylaminoethyl methacrylate) was synthesized by sequential living anionic polymerization in THF. The polymer has a numberaverage molecular weight of $75000 \mathrm{~g} \mathrm{~mol}^{-1}$ and was synthesized via sequential anionic polymerization. The weight fraction of PDMAEMA is $19 \%$. Details can be found elsewhere. ${ }^{32}$

The membranes were prepared via a non-solvent induced phase separation (NIPS) process. Solvents were filtered through $0.2 \mu \mathrm{m}$ PTFE filters before use. All glassware was cleaned using a $2: 1 \mathrm{H}_{2} \mathrm{SO}_{4} / \mathrm{H}_{2} \mathrm{O}_{2}$ mixture (diluted with $50 \%$ deionized water). Films were cast from a solution of $15 \mathrm{wt} \%$
$\mathrm{PS}_{81}-$ PDMAEMA $_{19}{ }^{75}$ in THF and DMF $(50: 50)$ solvent mixtures on a cleaned glass surface using a doctor blade ${ }^{33}$ with a step-height of $200 \mu \mathrm{m}$ at room temperature $\left(20{ }^{\circ} \mathrm{C}\right.$ in a humidity-controlled clean-room). After casting, the film was left in air for $90 \mathrm{~s}$ (open time) before immersing it in a bath containing deionized water (non-solvent). After $2 \mathrm{~h}$, the membranes were taken out from the water bath. The resulting membranes were self-supporting ${ }^{3}$ and were stored in Milli-Q water until they were used for the experiments. More details about the polymer and the membrane preparation procedures have been published elsewhere. ${ }^{33}$ In order to saturate the PDMAEMA chains and to avoid adsorption of the polymer beads to the domain walls, the membranes were immersed in a diluted silica nanoparticle solution (LUDOX SM-30 colloidal silica, 30\% suspension in water, received from Sigma-Aldrich) for 1-2 days. Next, the membranes were transferred to an aqueous solution of carboxy-functionalized polystyrene beads that were loaded with dye molecules and stayed there for 2-3 days. The concentration of the beads was less than nanomolar and their diameters were $(20 \pm 4)$, $(50 \pm 7)$, $(100 \pm 10),(200 \pm 10)$ and $(500 \pm 15) \mathrm{nm}$. The $20 \mathrm{~nm}$ sized beads (MoBiTech, GmbH, Germany) were loaded with nile red as the fluorescent dye, which has excitation and emission maxima around 535 and $575 \mathrm{~nm}$, respectively. The fluorophore in all other beads (PolySciences Europe $\mathrm{GmbH}$, Germany) was rhodamine. These beads featured excitation and emission maxima around 529 and $546 \mathrm{~nm}$, respectively. ${ }^{34}$ All solutions were prepared with Milli-Q water $(\sim \mathrm{pH} \quad 6)$ and the experiments were performed at room temperature $\left(20^{\circ} \mathrm{C}\right)$.

\section{Scanning electron microscopy (SEM)}

Field-emission SEM images of the membranes were taken on a LEO 1530 electron microscope (Zeiss). The samples were dried under vacuum overnight and were coated with a thin layer (approximately $2 \mathrm{~nm}$ ) of platinum prior to the imaging process.

\section{Single-particle tracking (SPT)}

The single-particle tracking experiments were carried out on a home-built fluorescence microscope. All the samples were excited with a diode laser (Monopower-532-100-SM, Alphalas) at $532 \mathrm{~nm}$. The excitation intensity was always $25 \mathrm{~W} \mathrm{~cm}^{-2}$. The excitation light was focused onto the sample with an oil immersion objective $(60 \times$, Olympus, NA $=1.45)$. The fluorescence from the polystyrene beads was collected through the same objective, long-pass filtered (LP 545 or LP 610, AHF Analysentechnik AG Tübingen, Germany), and detected with an EMCCD (iXon DV 887 DCS-BV, 512 pixel $\times 512$ pixel, Andor). The magnification of the microscope was 72 for the beads of $20 \mathrm{~nm}$ size and 148 for all other beads. The exposure time for recording a fluorescence image was $20 \mathrm{~ms}$. By fitting a Gaussian to the image of the bead the spatial location of the emitter could be determined with accuracies between 15-40 nm. The diffusion trajectories of the beads were obtained from a sequence of 1000-2000 consecutively recorded images, which were analyzed with the point tracking software ImageJ. ${ }^{35}$ 


\section{Results and discussion}

\section{Electron microscopy}

The morphology of the PS-PDMAEMA diblock copolymer membrane can be visualized by scanning electron microscopy. A top view of the membrane surface, which is the relevant surface for the experiments described here, is shown in Fig. 2a. SEM images of the bottom side as well as a cross-section of the membrane are presented in Fig. S1 of the ESI. $\dagger$ The domains feature predominantly circular profiles and only exceptionally ellipsoidal shapes are observed. The extensions of the domains along the $X$ and $Y$ axes in the laboratory frame have been determined with the software FIVE based on analySIS [Olympus Soft Imaging Solutions $\mathrm{GmbH}$ ]. As a general measure for the domain size we took the arithmetic mean of the extensions of the domains along the $X$ and $Y$ directions. It shows a wide distribution peaking at about $1000 \mathrm{~nm}$ with a width of $870 \mathrm{~nm}$ (FWHM), as shown in Fig. 2 b.

\section{Single-particle tracking of $20 \mathrm{~mm}$ beads}

Only a few of the beads of $20 \mathrm{~nm}$ size were adsorbed on the polymer matrix and appeared stationary during the experiment. Some other beads diffused in three dimensions manifested by the appearance and disappearance of the beads in the focal plane of the microscope. Yet most of the beads, about $80 \%$, showed diffusion in two dimensions and examples for such trajectories are shown in Fig. 3.

The trajectories reflect the confined diffusion of the beads in domains that differ from each other with respect to size and shape. While some trajectories fill a circular area (a and c) others cover an elliptical area ( $\mathrm{d}$ and e). Also the maximum
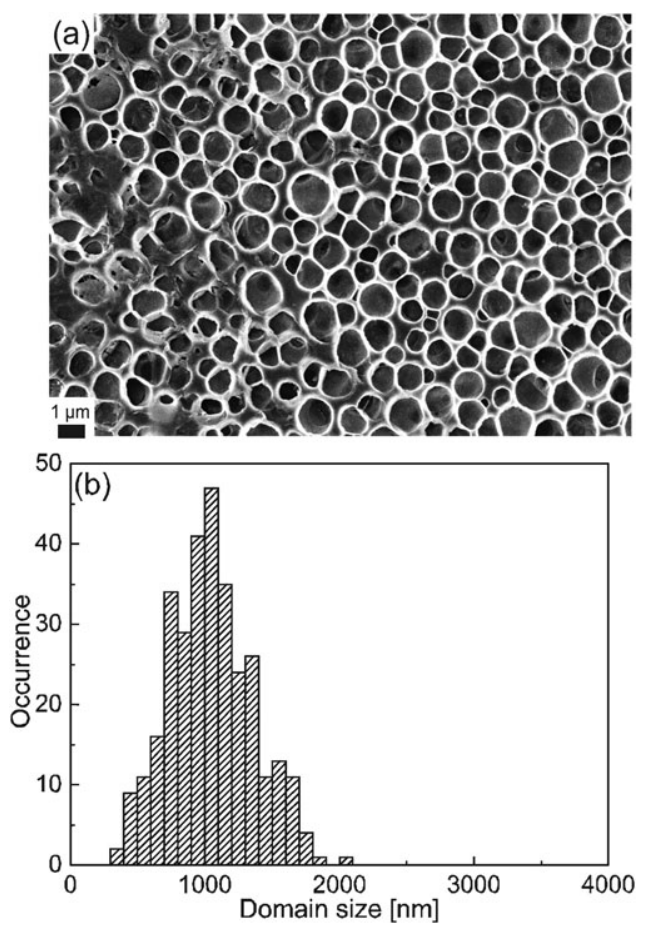

Fig. 2 (a) SEM image of the PS-PDMAEMA diblock copolymer membrane. The scale bar corresponds to $1 \mu \mathrm{m}$. (b) Domain size distribution as determined from SEM imaging.

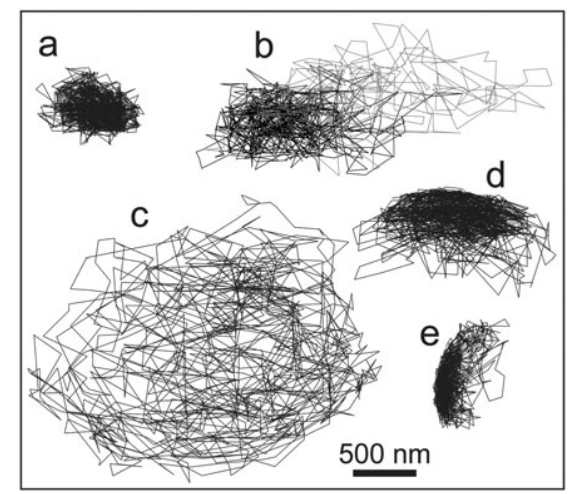

Fig. 3 Examples of diffusion trajectories from individual $20 \mathrm{~nm}$ sized polystyrene beads. The trajectories are numbered $(\mathrm{a}-\mathrm{e})$ for reference. The scale bar corresponds to $500 \mathrm{~nm}$ and is valid for all traces.

extensions of the space covered by the trajectories varied. It covered the range from $100 \mathrm{~nm}$ to $3500 \mathrm{~nm}$. In a few cases it was observed that the bead left the initial domain, Fig. 3b, and moved to a neighbouring domain. In the ESI $\dagger$, movies of the trajectories a (movie $\mathrm{S} 1$ ) and $\mathrm{b}$ (movie $\mathrm{S} 2$ ) are provided. In order to analyze the trajectories more quantitatively, we calculated the mean-square displacement (MSD) of the beads according to

$$
\begin{aligned}
\operatorname{MSD} & =\left\langle(X(n)-X(n+i))^{2}+(Y(n)-Y(n+i))^{2}\right\rangle \\
& =\left\langle(\Delta X(i))^{2}+(\Delta Y(i))^{2}\right\rangle .
\end{aligned}
$$

Here, $X$ and $Y$ are the coordinates of the bead in the laboratory frame perpendicular to the optical axis, $n$ is the frame number of the image on the CCD, $i$ is the increment of the frame number corresponding to the time lag between the two exposures, and the brackets denote the averaging with respect to $n$. For two examples, corresponding to the trajectories a and $\mathrm{d}$ in Fig. 3, the MSD versus time lag is plotted in Fig. 4.

The data points show $\langle(\Delta X)\rangle^{2}$ and $\langle(\Delta Y)\rangle^{2}$ separately for the $X$ and $Y$ directions as well as their sum according to eqn (1). For the first few data points all time lag curves show a linear increase of the MSD as a function of time, see insets in Fig. 4, and level off to a constant value for larger time lags, which is caused by the confinement of the diffusion of the polystyrene beads to the domains of the membrane. The linear extensions of the domains in $X$ and $Y$ directions, $L_{x}$ and $L_{y}$, can be obtained from ref. 36

$$
\begin{aligned}
& \left\langle(\Delta X(t))^{2}\right\rangle=\frac{L_{x}^{2}}{6}-\frac{16 L_{x}^{2}}{\pi^{4}} \sum_{n=1(\text { odd })}^{\infty} \frac{1}{n^{4}} \exp \left\{-\left(\frac{n \pi}{L_{x}}\right)^{2} D_{x} t\right\} \\
& \left\langle(\Delta Y(t))^{2}\right\rangle=\frac{L_{y}^{2}}{6}-\frac{16 L_{y}^{2}}{\pi^{4}} \sum_{n=1 \text { (odd })}^{\infty} \frac{1}{n^{4}} \exp \left\{-\left(\frac{n \pi}{L_{y}}\right)^{2} D_{y} t\right\}
\end{aligned}
$$

where $D_{x}$ and $D_{y}$ are the one-dimensional diffusion coefficients along $X$ and $Y$. The full lines in Fig. 4 correspond to fits of the data to these equations, which yield excellent agreement with the experiment and allow extracting $L_{x}$ and $L_{y}$ from the MSD curves. For the example shown in Fig. $4 \mathrm{a}$, the fits yield $L_{x}=(532 \pm 6) \mathrm{nm}, L_{y}=(569 \pm 9) \mathrm{nm}$ for the extensions 

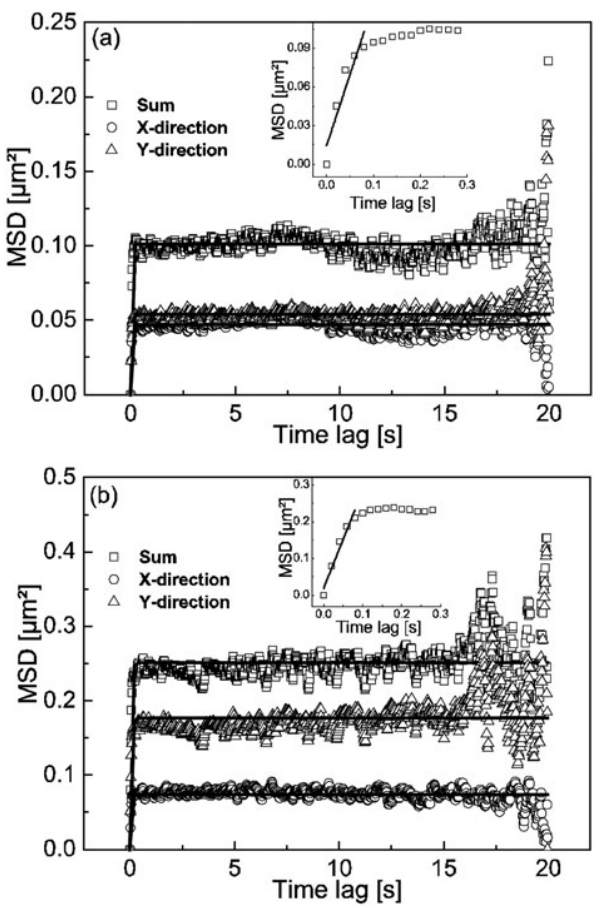

Fig. 4 MSD versus time lag for a domain of (a) (nearly) circular shape and (b) a domain of elliptical shape. The data points are given separately for the diffusion along the $X$ direction (circles), $Y$ direction (triangles), and their sum according to eqn (1) (squares). The full lines are fits to the data according to eqn (2) and (3). The insets show the linear increase of the MSD for small time lags on an enlarged scale.

in $X$ and $Y$ directions, and $L_{\mathrm{D}}=(779 \pm 10) \mathrm{nm}$ for the summed MSD. The latter value is in perfect agreement with $L_{\mathrm{D}}=\sqrt{L_{x}^{2}+L_{y}^{2}}$ which reproduces the same number. The small difference between $L_{x}$ and $L_{y}$ reflects the almost circular shape of the area covered by the diffusion of the respective fluorescent bead (Fig. 3, trajectory a). For the example shown in Fig. $4 b$ the respective numbers obtained from the fits are $L_{x}=(666 \pm 10) \mathrm{nm}, L_{y}=(1030 \pm 29) \mathrm{nm}$, and $L_{\mathrm{D}}=(1226 \pm 26) \mathrm{nm}\left(L_{\mathrm{D}}=\sqrt{L_{x}^{2}+L_{y}^{2}}=1226 \mathrm{~nm}\right)$ reproducing the elliptical shape of the domain (Fig. 3, trajectory d).

In order to compare the domain sizes from SPT with those from SEM we have to consider that $L_{\mathrm{D}}$ represents the length of the diagonal of the rectangle that encloses the diffusion trajectory. For the comparison, therefore, a better measure of the domain sizes is provided by the arithmetic mean $L_{\mathrm{M}}=\frac{1}{2}\left(L_{x}+L_{y}\right)$. The distribution of this parameter for the $20 \mathrm{~nm}$ sized beads is shown in Fig. 5. It features a maximum at $650 \mathrm{~nm}$ and a width of $780 \mathrm{~nm}$ (FWHM). For comparison, the previously obtained domain size distribution from SEM is displayed in Fig. 5 as well.

Obviously, the distribution obtained from SPT is shifted by about $350 \mathrm{~nm}$ towards lower values with respect to the distribution obtained from SEM. We ascribe this discrepancy to the fact that both methods, SEM and SPT, monitor entirely different properties. The contrast observed in electron microscopy images reflects the differences in the ionization potentials of the materials under study. Neither the reduction of the domain diameter by about $30 \mathrm{~nm}$ due to decorating the

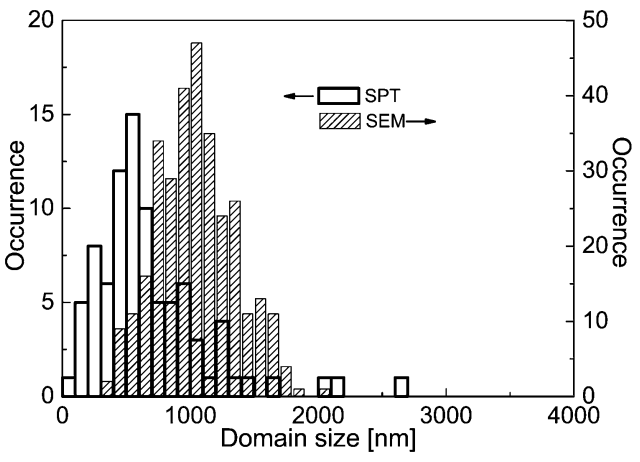

Fig. 5 Comparison of the domain size distributions from SPT of $20 \mathrm{~nm}$ sized polystyrene beads (white) and from SEM (shaded). The left scale is valid for the SPT data and the right scale is valid for the SEM data.

domain walls with the PDMAEMA chains, nor the repulsive forces between the PDMAEMA chains and the carboxylfunctionalized dye loaded polystyrene bead probes, nor the hydrodynamic hindrance of the diffusion due to wall effects ${ }^{37}$ can be visualized with this method. However, these interactions have a crucial influence on the diffusion behaviour of the probe particles and become observable in the SPT experiments. Furthermore, from the linear increase of the MSD curves, see insets in Fig. 4, we determined the local diffusion coefficients for Brownian motion in two dimensions according to

$$
\mathrm{MSD}=4 D t .
$$

Here $D$ denotes the diffusion coefficient, which is related to the one-dimensional diffusion coefficients by $4 D=2 D_{x}+2 D_{y}{ }^{36}$
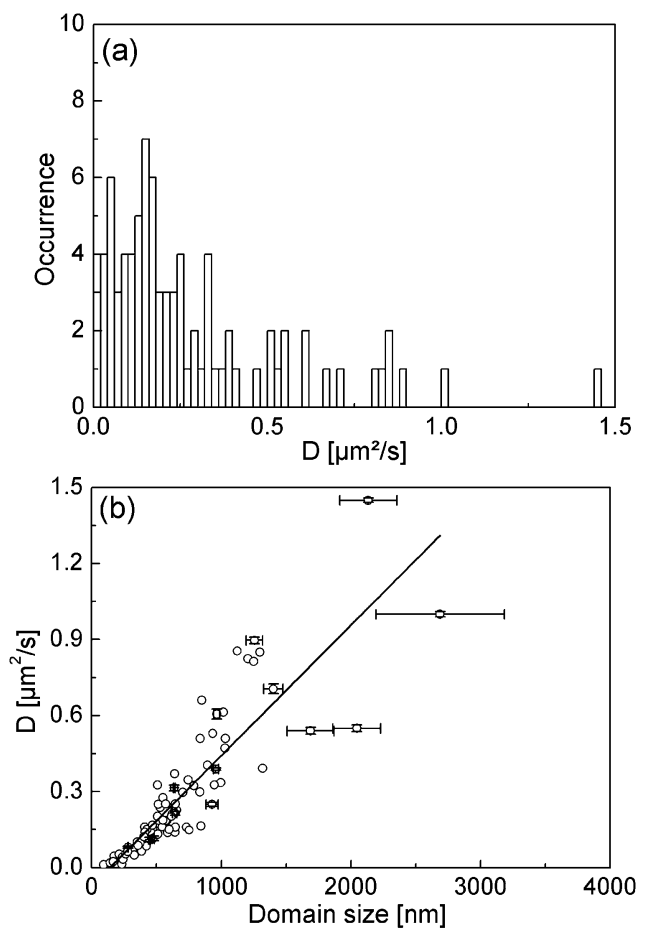

Fig. 6 (a) Distribution of the diffusion coefficient from SPT of the $20 \mathrm{~nm}$ sized beads. (b) Diffusion coefficient as a function of domain size (circles). The full line corresponds to a linear fit to the data with a correlation coefficient of 0.87 . 
We want to note that the values of $D_{x}$ and $D_{y}$ cannot be determined with good accuracy from the fits of the MSD curves due to the restricted diffusion. Therefore we evaluated the diffusion coefficient $D$ from the linear increase of the first few data points of the time lag curves of the summed MSD. Doing so results in a broad distribution of diffusion coefficients, as shown in Fig. 6a.

From the histogram we find a value of $0.28 \mu \mathrm{m}^{2} \mathrm{~s}^{-1}$ for the mean of the diffusion coefficient, which is in the same order of magnitude with data found for similar systems. ${ }^{24}$ The broad distribution that has been found for the diffusion coefficients testifies a large degree of heterogeneity within the membrane material. Interestingly, the actual magnitude of the diffusion coefficient is linearly correlated with the size of the domain in which the polymer bead is diffusing, Fig. 6b. The solid line in Fig. $6 \mathrm{~b}$ represents a linear fit to the data points corresponding to a correlation coefficient of $0.87 \pm 0.02$. This observation is in general agreement with the fact that for confined diffusion, hydrodynamic wall effects lead to a significant reduction of the diffusion coefficient. ${ }^{37}$ This finding further substantiates our conjecture that the differences in the domain size distributions from SEM and SPT reflect the influence of the walls of the domains on the diffusion behaviour of the probe beads.

\section{Diffusion of larger beads}

In order to investigate the influence of the size of the beads on the results we repeated the experiments described above with beads that were 50, 100, 200 and $500 \mathrm{~nm}$ in diameter. In Fig. 7
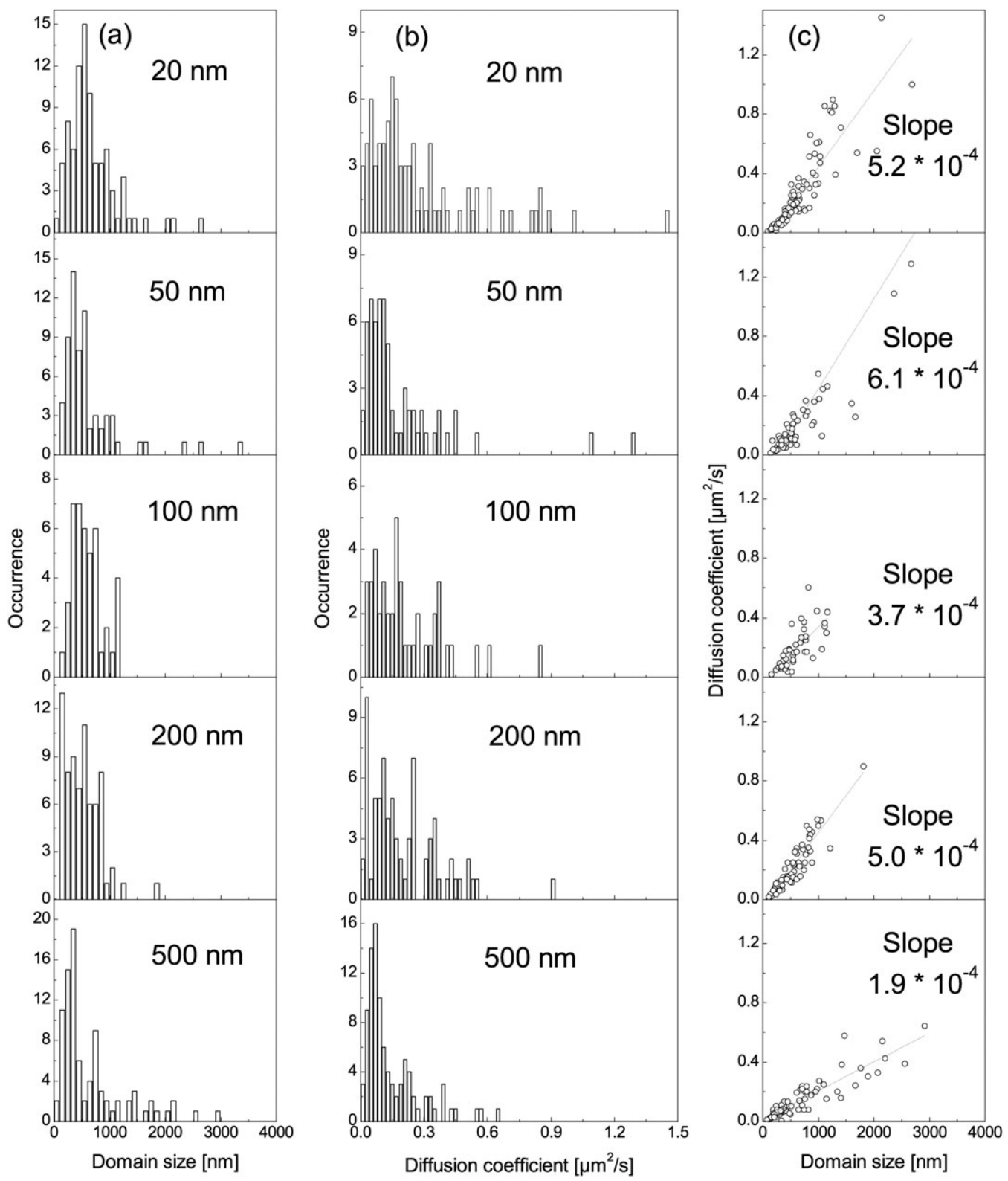

Fig. 7 (a) Distributions of the domain size, (b) the diffusion coefficients, and (c) correlation between the diffusion coefficient and the domain size as a function of the size of the probe beads, which increases from top to bottom. The full lines in (c) correspond to linear fits to the data and the respective slopes are given in the figures. For one of the probes of $50 \mathrm{~nm}$ size (diffusing in a domain of $3.4 \mu \mathrm{m}$ diameter) we found a diffusion coefficient of $2.8 \mu \mathrm{m}^{2} \mathrm{~s}^{-1}$. These data points are not shown for scaling reasons but have been included in the analysis. 
Table 1 Median of the domain size, median of the diffusion coefficient and slope of the correlation between the diffusion coefficient and the domain size for the data shown in Fig. 7

\begin{tabular}{llll}
\hline & $\begin{array}{l}\text { Median, } \\
\text { domain size/nm } / \mathrm{nm}\end{array}$ & $\begin{array}{l}\text { Median, diffusion } \\
\text { coefficient } \\
D / \mu \mathrm{m}^{2} \mathrm{~s}^{-1}\end{array}$ & $\begin{array}{l}\text { Slope of } D \text { versus } \\
\text { domain size } \\
\left(\mu \mathrm{m}^{2} \mathrm{~s}^{-1} \times \mathrm{nm}\right)\end{array}$ \\
\hline 20 & 571 & 0.185 & $5.2 \times 10^{-4}$ \\
50 & 438 & 0.112 & $6.1 \times 10^{-4}$ \\
100 & 549 & 0.169 & $3.7 \times 10^{-4}$ \\
200 & 498 & 0.160 & $5.0 \times 10^{-4}$ \\
500 & 387 & 0.091 & $1.9 \times 10^{-4}$ \\
\hline
\end{tabular}

the obtained distributions for the domain sizes, Fig. 7a, the diffusion coefficients, Fig. 7b, and the correlation between the diffusion coefficients and the domain sizes, Fig. 7c, are shown as a function of the diameter of the beads. However one would expect that the diffusion coefficient levels off as a function of the domain size if the particle diameter becomes very small with respect to the domain size. Remarkably even for ratios of the particle size/domain size in the range of 0.01 the linear correlation between domain size and the diffusion coefficient is still observable.

For a better comparison, the data from the $20 \mathrm{~nm}$ sized beads are reproduced in this figure as well. In Table 1 we have summarized the median of the distributions for the domain size and the diffusion coefficient as well as the slopes of their linear correlation as a function of the bead size.

For the beads with diameters between 20 and $100 \mathrm{~nm}$, the data do not show statistically significant deviations from each other. Yet, for the beads of $200 \mathrm{~nm}$ and $500 \mathrm{~nm}$ in diameter, both the domain size distribution and the distribution of the diffusion coefficients are shifted to even smaller values with respect to the histograms obtained for the smaller beads. This is also reflected in a significant reduction of the slope of the correlation curve for the $500 \mathrm{~nm}$ sized beads. This finding is not too surprising and it could have been anticipated that hydrodynamic wall effects will significantly affect the diffusion behaviour of beads with a diameter in the order of $20-50 \%$ of the domain diameter.

\section{Conclusions}

We studied the material transport in nanoporous polystyrene-block-poly(2-dimethylaminoethyl methacrylate) ( $\mathrm{PS}_{81^{-}}$ PDMAEMA $_{19}{ }^{75}$ ) membranes by SPT of dye-loaded carboxyfunctionalized polystyrene beads as a function of the size of the single-particle probes. From these experiments we determined the domain size distribution of the membranes and the local diffusion coefficient. It turned out that the data did not depend on the size of the probe particles as long as the ratio of particle/domain size did not exceed a value of about 0.1 . For larger ratios of the particle size/domain size the distributions of the domain size (diffusion coefficient) do not reproduce those obtained at smaller ratios, reflecting that separation as a function of particle size becomes effective. Generally, the domain size distributions obtained from SPT yielded systematically smaller domains with respect to a reference experiment exploiting SEM. It is likely that the main cause for this discrepancy stems from the interaction of the probe particles with the domain walls. This assignment is in line with the observation of even stronger deviations for the larger beads. Since material transport by diffusion is an essential feature of a membrane, the domain size distribution obtained from SPT should be considered to reveal the effective domain sizes that are relevant for the function of the membrane.

\section{Acknowledgements}

We gratefully acknowledge financial support from the VolkswagenStiftung, Hannover, within the framework of the programme "Complex Materials". Tobias Rudolph is acknowledged for help during membrane preparation. Further we thank Werner Köhler for fruitful discussions about diffusion and Florian Spreitler for assistance with LabVIEW programming.

\section{References}

1 M. J. Pellin, P. C. Stair, G. Xiong, J. W. Elam, J. Birrell, L. Curtiss, S. M. George, C. Y. Han, L. Iton, H. Kung, M. Kung and H.-H. Wang, Catal. Lett., 2005, 102, 127.

2 N. M. Islam, M. Chatterjee, Y. Ikushima, T. Yokoyama and H. Kawanami, Int. J. Mol. Sci., 2010, 11, 164.

3 F. Schacher, M. Ulbricht and A. H. E. Müller, Adv. Funct. Mater., 2009, 19, 1040.

4 B. Sea and K.-H. Lee, Bull. Korean Chem. Soc., 2001, 22, 1400.

5 H.-J. Lee, H. Suda, K. Haraya and S.-H. Moon, J. Membr. Sci., 2007, 296, 139.

6 J. C. T. Eijkel and A. van den Berg, Microfluid. Nanofluid., 2005, 1, 249.

7 G. Nunnery, E. Hershkovits, A. Tannenbaum and R. Tannenbaum, Langmuir, 2009, 25, 9157.

8 S. Song, A. K. Singh, T. J. Shepodd and B. J. Kirby, Anal. Chem., 2004, 76, 2367.

9 W. H. Fissell, A. Dubnisheva, A. N. Eldridge, A. J. Fleischman, A. L. Zydney and S. Roy, J. Membr. Sci., 2009, 326, 58.

10 T. Lebold, C. Jung, J. Michaelis and C. Bräuchle, Nano Lett., 2009, 9, 2877.

11 T. Hoare, J. Santamaria, G. F. Goya, S. Irusta, D. Lin, S. Lau, R. Padera, R. Langer and D. S. Kohane, Nano Lett., 2009, 9, 3651.

12 S. Rols, H. Jobic and S. Helmet, C. R. Phys., 2007, 8, 777.

13 H. Jobic, A. I. Skoulidas and D. S. Sholl, J. Phys. Chem. B, 2004, 108, 10613.

14 S. Vasenkov, W. Böhlmann, P. Galvosas, O. Geier, H. Liu and J. Kärger, J. Phys. Chem. B, 2001, 105, 5922.

15 S. Vasenkov, K. Ulrich, C. Selle, J. Kärger and J. Käs, Diffus. Fundam., 2005, 2, 136.1.

16 Y. Fu, F. Ye, W. G. Sanders, M. M. Collinson and D. A. Higgins, J. Phys. Chem. B, 2006, 110, 9164.

17 S. M. Mahurin, S. Dai and M. D. Barnes, J. Phys. Chem. B, 2003, 107, 13336.

18 M. J. Saxton and K. Jacobson, Annu. Rev. Biophys. Biomol. Struct., 1997, 26, 373.

19 V. Levi and E. Gratton, Cell Biochem. Biophys., 2007, 48, 1.

20 C. Joo, H. Balci, Y. Ishitsuka, C. Buranachai and T. Ha, Аnnu. Rev. Biochem., 2008, 77, 51.

21 K. Ritchie and J. Spector, Biopolymers, 2007, 87, 95.

22 J. Kirstein, B. Platschek, C. Jung, R. Brown, T. Bein and C. Bräuchle, Nat. Mater., 2007, 6, 303.

23 C. Jung, J. Kirstein, B. latschek, T. Bein, M. Budde, I. Frank, K. Müllen, J. Michaelis and C. Bräuchle, J. Am. Chem. Soc., 2008, 130, 1638.

24 M. Yorulmaz, A. Kiraz and A. L. Demirel, J. Phys. Chem. B, 2009, 113, 9640 .

25 C. Hellriegel, J. Kirstein, C. Bräuchle, V. Latour, T. Pigot, R. Olivier, S. Lacombe, R. Brown, V. Guieu, C. Payrastre, A. Izquierdo and P. Mocho, J. Phys. Chem. B, 2004, 108, 14699. 
26 C. Hellriegel, J. Kirstein and C. Bräuchle, New J. Phys., 2005, $7,23$.

27 Th. Schmidt, G. J. Schütz, W. Baumgartner, H. J. Gruber and H. Schindler, Proc. Natl. Acad. Sci. U. S. A., 1996, 93, 2926.

28 C. Seebacher, C. Hellriegel, F.-W. Deeg and C. Bräuchle, J. Phys. Chem. B, 2002, 106, 5591.

29 A. Zürner, J. Kirstein, M. Döblinger, C. Bräuchle and Th. Bein, Nature, 2007, 450, 705.

30 M. Ulbricht, Polymer, 2006, 47, 2217.

31 F. A. Plamper, M. Ruppel, A. Schmalz, O. Borisov, M. Ballauff and A. H. E. Müller, Macromolecules, 2007, 40, 8361.
32 F. Schacher, M. Müllner, H. Schmalz and A. H. E. Müller, Macromol. Chem. Phys., 2009, 210, 256.

33 F. Schacher, T. Rudolph, F. Wieberger, M. Ulbricht and A. H. E. Müller, ACS Appl. Mater. Interfaces, 2009, 1, 1492.

34 http://www.polysciences.com/Catalog/Department/81/ categoryId_341/.

35 I. F. Sbalzarini and P. Koumoutsakos, J. Struct. Biol., 2005, 151, 182.

36 A. Kusumi, Y. Sako and M. Yamamoto, Biophys. J., 1993, 65, 2021

37 J. M. Nitsche and G. Balgi, Ind. Eng. Chem. Res., 1994, 33, 2242. 